\title{
Electron microscopic studies of human haemosiderin and ferritin
}

\author{
MP WEIR, GA SHARP, TJ PETERS \\ From the Division of Clinical Cell Biology, Medical Research Council Clinical Research Centre, Harrow, \\ Middlesex
}

SUMMARY Ferritin and haemosiderin were isolated from fresh frozen human spleens that had been removed from patients with secondary iron overload due to multiple transfusions. Haemosiderin was solubilised by a novel technique that maintains its integrity. Unstained preparations of haemosiderin and ferritin were visualised and quantitative measurements made of the volumes of iron core. The mean diameter of the ferritin core $(6.4 \mathrm{~nm})$ was larger than that of haemosiderin $(5.7 \mathrm{~nm})$. In addition, haemosiderin, in contrast to ferritin, showed a large number of cores of less than $5 \mathrm{~nm}$ in diameter. Negatively stained preparations of haemosiderin and ferritin were visualised, confirming the small core size of the haemosiderin. The protein shell of haemosiderin, unlike that of ferritin, was thinner and irregular. These findings are consistent with the suggestion that haemosiderin is derived from ferritin by partial proteolysis and partial solubilisation of the iron core, presumably by lysosomal action.

Haemosiderin is a complex of iron oxyhydroxide microcrystals with protein, phosphate, some metal ions, and, perhaps, lipids. ${ }^{2}$ It is the principal protein that stores iron in conditions of iron overload such as $\beta$ thalassaemia and may be responsible for the severe damage to tissue that accompanies such syndromes. ${ }^{3}$ Its lysosomal location, radioactive iron pulse-chase experiments, ${ }^{4}$ and close physiochemical similarities with both haemosiderin crystallites and ferritin crystallites ${ }^{5}$ has led to the view that it is a breakdown product of ferritin, but there is no direct evidence for this.

In contrast to ferritin, in which the individual crystallites (termed cores) are enclosed and solubilised by a protein shell, haemosiderin cores form an insoluble aggregate, which makes them difficult to examine morphologically. We have used a method that has recently been developed to solubilise and disaggregate haemosiderin ${ }^{2}$ and will therefore facilitate such studies. Negative staining techniques were used to look for possible remnants of the ferritin protein shell in the purified preparations of haemosiderin.

\section{Material and methods}

ISOLATION OF HAEMOSIDERIN AND FERRITIN Haemosiderin and ferritin were isolated from spleens overloaded with iron from patients who had received multiple transfusions for $\beta$ thalassaemia and frozen fresh to $-20^{\circ} \mathrm{C}$. Haemosiderin was isolated by centrifugation through concentrated potassium iodide solutions ${ }^{2}$ and was solubilised in $0.02 \mathrm{M}$ tetramethylammonium hydroxide, $\mathrm{pH} 12 \cdot 3$, by gentle stirring for 45 minutes at room temperature. Ferritin was purified according to the method of Huebers et al. ${ }^{6}$

\section{ELECTRON MICROSCOPY}

Samples of solubilised haemosiderin were applied directly to mesh 400 copper grids coated with carbon (Athene Ltd, United Kingdom) and viewed under a Philips EM 300 microscope operating at $100 \mathrm{KV}$. Samples of haemosiderin for negative staining were applied as a sonicated suspension in water and then stained with potassium phosphotungstate, $\mathrm{pH} 6.5$, for 30 seconds, dried in air, and viewed under the electron microscope. Ferritin was applied to grids as an aqueous solution then viewed directly or, after negative staining, with potassium phosphotungstate, $\mathrm{pH} 6.5$. Diameters of 
particles of 100 to 150 cores for every sample were measured from micrographs. The volume of the particles was estimated, assuming that the shape was spherical.

\section{Results and discussion}

Fig. 1 shows an electron micrograph of a section of human spleen loaded with iron and stained with uranyl acetate and lead citrate. The figure shows the morphological definition of haemosiderin as large, densely packed aggregates of iron cores. The insert at high power shows that these aggregates were bounded by a membrane, presumably lysosomal. ${ }^{7}$ Individual ferritin cores were seen throughout the cytoplasm.

To permit closer inspection of the cores of haemosiderin, granules were isolated, solubilised, and examined unstained (Fig. 2). Comparison with ferritin (Fig. 3) showed clearly that the cores of haemosiderin were smaller and more irregular in shape, confirming the results of Fischbach et al. ${ }^{8}$ This observation is not an artefact of the method for solubilisation: inspection of a suspension of haemosiderin in water showed mostly large aggregates, but well separated cores could be clearly seen (Fig. 4). Fig. 5 shows the distributions of core size estimated from unstained electron micrographs of ferritin and haemosiderin.
The diameter of average volume (assuming spherical shape) for haemosiderin was about $5.7 \mathrm{~nm}$, compared with $6.4 \mathrm{~nm}$ for ferritin, indicating that the cores of haemosiderin were, on average, $70 \%$ of the volume of ferritin cores. The distribution of the core size of haemosiderin was broader and lacking as clear a cut off (above $7 \cdot 0 \mathrm{~nm}$ ) as ferritin. There were many cores in haemosiderin below $5 \mathrm{~nm}$ in diameter, whereas there were very few such cores in ferritin, which gave the distribution of particle size in haemosiderin a strong asymmetric and almost bimodal appearance. Such a wide distribution could result from progressive partial chemical degradation of ferritin cores to form haemosiderin, but with a continuous addition of undegraded ferritin providing the larger cores. This, of course, implies that iron is being released from haemosiderin in the lysosome in the form of soluble complexes, which could therefore implicate haemosiderin in the peroxidation of iron catalysed lipid observed in iron overload $^{9-11}$ and possibly in the disruption of lysosomes after autolysis. ${ }^{1213}$

We have shown that the cores of haemosiderin are smaller and more aggregated than those of ferritin. Both of these features may be a direct result of degradation of the protein shell of ferritin as this would allow greater access for reducing or chelating agents and would also cause self association of cores. Negative staining of haemosiderin shows incomplete

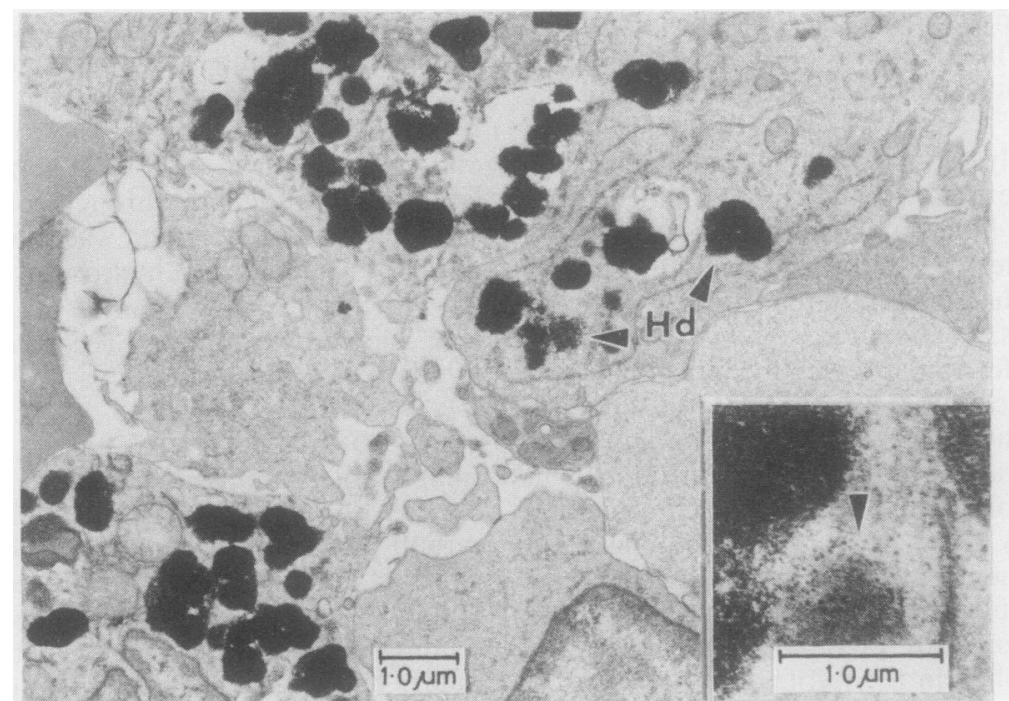

Fig. 1 Haemosiderin granules seen as aggregates of cores packed together to varying degrees within section of human spleen loaded with iron and stained with uranyl acetate and lead citrate. $\times 7980$. Insert of high power shows that individual cores of ferritin (diameter $\alpha 7 \mathrm{~nm}$ ) (arrowed) are scattered through cytoplasm of spleen. 


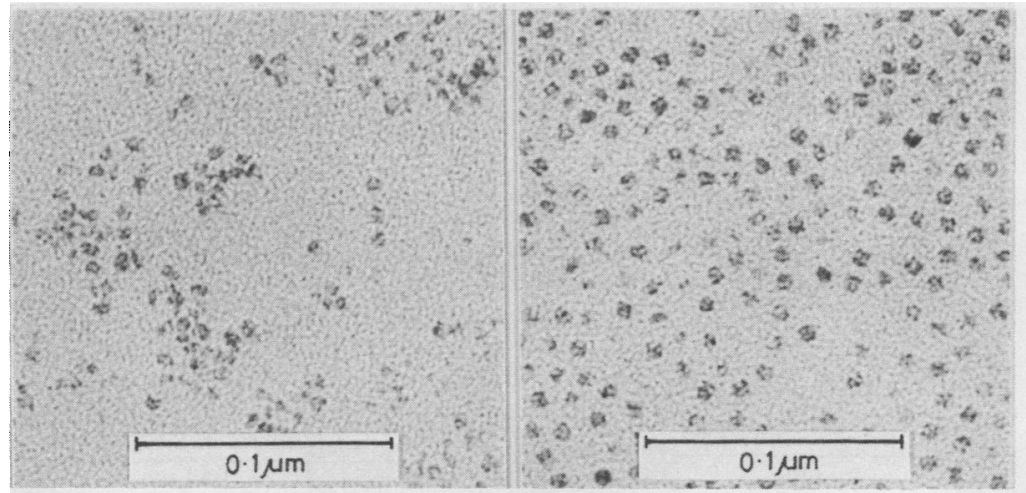

Fig. 2 Haemosiderin applied to grid as solution in tetramethylammonium hydroxide. $\times 255000$.

Fig. 3 Ferritin, isolated from same spleen as haemosiderin in Fig. 2, applied as an aqueous solution to grid. $\times 255000$.

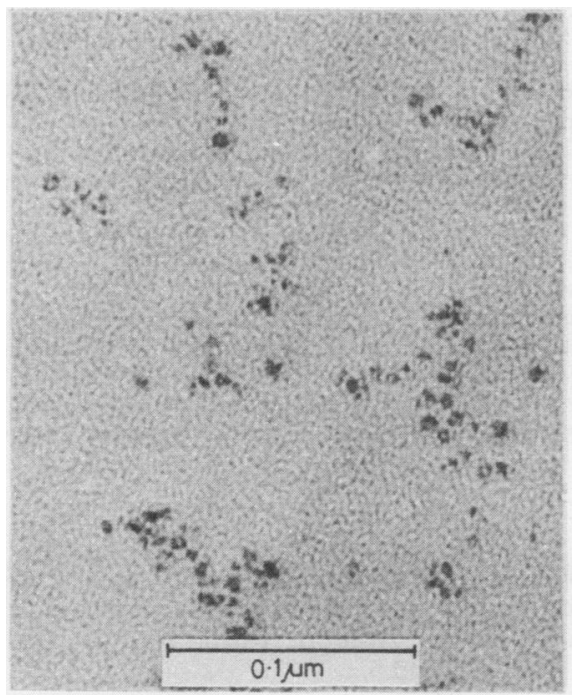

Fig. 4 Haemosiderin applied to grid as water suspension. $\times 255000$.
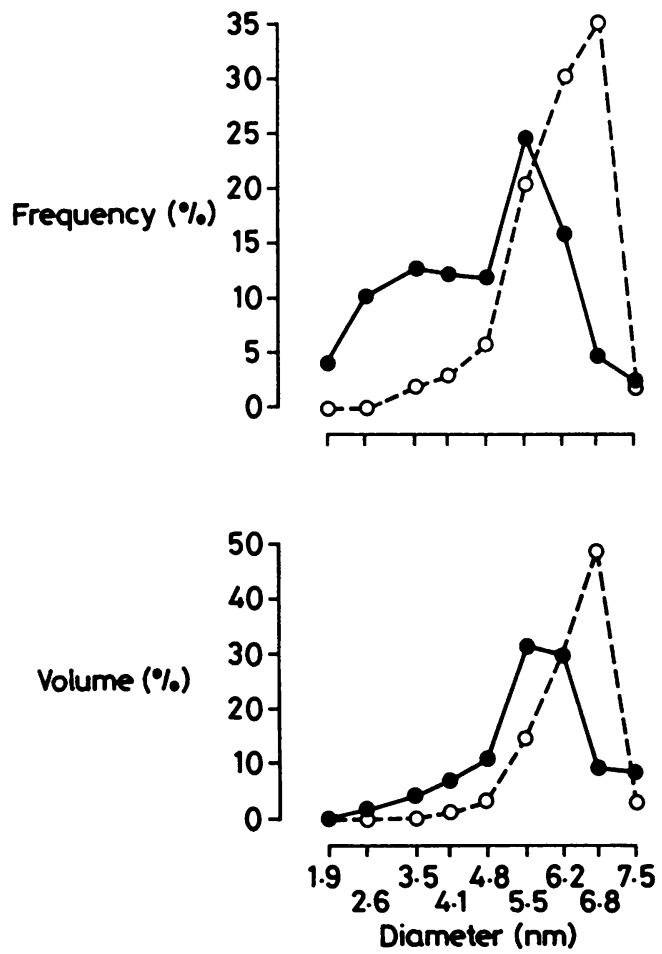

Fig. 5 Distributions of frequency and volume of haemosiderin in tetramethylammonium hydroxide solution (filled circles) and ferritin (open circles). 


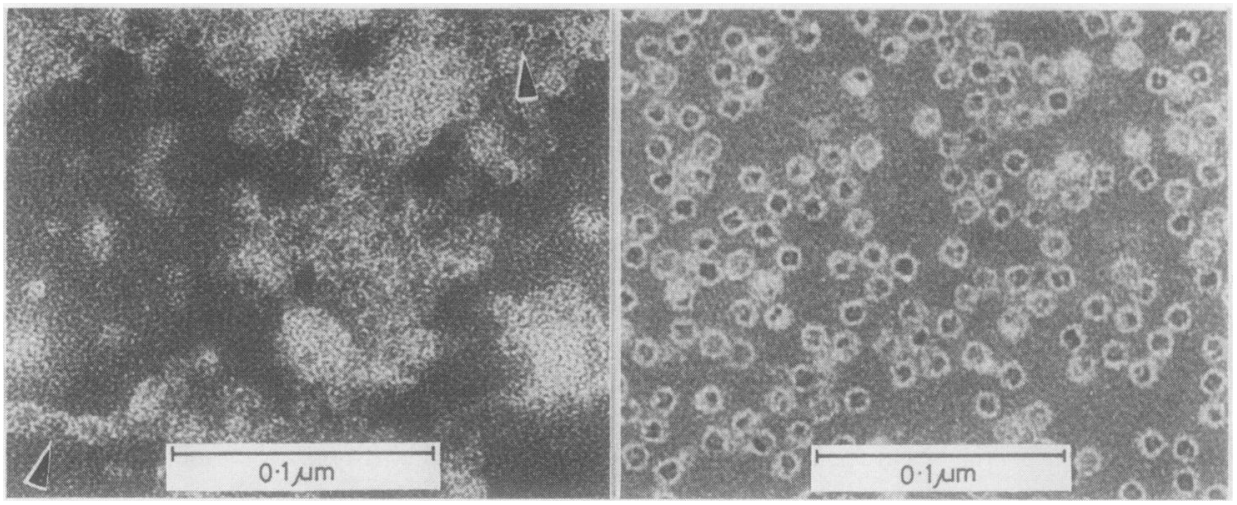

Fig. 6 Negatively stained haemosiderin. Variable presence of non-staining material around cores is indicated by arrows. $\times 255000$.

Fig. 7 Negatively stained ferritin showing uniform, complete non-staining material around iron cores. Iron cores also have larger diameter than those of haemosiderin shown in Fig. $6 . \times 255000$.

rings of protein around the cores (Fig. 6) compared with the complete protein shell of ferritin (Fig. 7), which agrees with other evidence that this protein is derived from ferritin. ${ }^{2}$

We thank Ms H Grindley for technical help and Drs DJ Gibson and D Dixon for helpful discussions. MPW was in receipt of a research studentship from the Medical Research Council.

\section{References}

' McKay RH, Fineberg RA. Horse spleen haemosiderin. I. Isolation. Arch Biochem Biophys 1964;104:487-95.

${ }^{2}$ Weir MF, Gibson JF, Peters TJ. Biochemical studies on the isolation and characterization of human spleen haemosiderin. Biochem J 1984;223:31-8.

${ }^{3}$ Selden C, Owen H, Hopkins JMP, Peters TJ. Studies on the concentrations of iron proteins in liver biopsy specimens from patients with iron overload with special reference to the rate in lysosomal disruption. Br J Haematol 1980;44:593-603.

${ }^{4}$ Shoden A, Gabrio BW, Finch CA. The relationship between ferritin and haemosiderin in rabbits and man. $J$ Biol Chem 1953;204:823-30.

s Bell SH, Weir MP, Dickson DPE, Gibson JF, Peters TJ. Mössbauer spectroscopic studies of haemosiderin and ferritin. Biochim Biophys Acta 1984;787:227-36.
${ }^{\circ}$ Huebers H, Huebers E, Crichton RR. Isolation and characterisation of rat mucosal ferritin. FEBS Letts 1974; 44:302-8.

${ }^{7}$ Richter GW. Studies of iron overload rat liver sidersome formation. Lab Invest 1984; 50:26-35.

${ }^{8}$ Fischbach FA, Gregory DW, Harrison PM, Hoy TG, Williams JM. Structure of haemosiderin and its relationship to ferritin. J Ultrastruct Res 1971;37:495-503.

' Heys AD, Dormandy TL. Lipid peroxidation in iron-loaded spleens. Clin Sci 1981;60:295-301.

${ }^{10} \mathrm{O}$ 'Connell MJ, Ward R, Baum H, Peters TJ. The role of haemosiderin in iron-mediated membrane lipid peronidation. Life Chemistry Reports (in press).

" O'Connell MJ, Ward R, Baum H, Peters TJ. The role of iron in ferritin and haemosiderin-mediated lipid peronidation in lysosomes. Biochem J (in press).

${ }^{12}$ Peters TJ, Selden C, Seymour CA. Lysosomal disruption in the pathogenesis of hepatic damage in primary and secondary haemochromatosis. Ciba Found Symp 1977;NS51:317-25.

${ }^{13}$ Seymour CA, Peters TJ. Organelle pathology in primary and secondary haemochromatosis. Br J Haematol 1979;40:23953.

Requests for reprints to: Professor TJ Peters, Division of Clinical Cell Biology, Medical Research Council Clinical Research Centre, Watford Road, Harrow, Middlesex, HA1 3UJ, England. 